\title{
Manejo da cultura do melão submetida a frequências de irrigação e fertirrigação com nitrogênio
}

\author{
André R Campelo; Benito M Azevedo; Joaquim R Nascimento Neto; Thales VA Viana; Luís G Pinheiro \\ Neto; Rodrigo H Lima
}

UFC-Depto. Eng. Agrícola, C. Postal 12168,60455-970 Fortaleza-CE; andre rcampelo@hotmail.com.br; benitoazevedo@hotmail.com; netoparaguai456@yahoo.com.br; thales@ufc.br; netolgp@hotmail.com; rodrigolima@agronomo.eng.br

\section{RESUMO}

O melão é a principal olerícola exportada pelo estado do Ceará, sendo uma importante geradora de renda e empregos para a região. Diante do exposto, esse trabalho objetivou avaliar os efeitos de diferentes frequências de irrigação e de fertirrigação com nitrogênio sobre características físicas e a produtividade do meloeiro amarelo. O experimento foi conduzido no município de Cruz-CE, de 10 de outubro a 15 de dezembro de 2010. A cultura utilizada foi o melão, híbrido Canarian Kobayashi, irrigada por gotejamento. No experimento I foram avaliadas seis frequências de irrigação: diária $(50 \%$ de manhã e $50 \%$ de tarde); diária (100\% pela manhã); diária ( $100 \%$ de tarde); a cada dois dias pela manhã; a cada três dias pela manhã e a cada quatro dias pela manhã. No experimento II foram avaliadas seis frequências de fertirrigação com nitrogênio: duas $(2 \mathrm{~F})$; quatro (4F); oito (8F); dezesseis (16F); trinta e duas (32F) e sessenta e quatro (64F) fertirrigações durante o ciclo de desenvolvimento da cultura. Foi empregado o delineamento experimental em blocos casualizados com quatro repetições. Realizou-se também análise econômica simplificada para os dois experimentos. No primeiro experimento, os valores de massa dos frutos, produtividade comercial, diâmetro polar, diâmetro equatorial e espessura da polpa sofreram influência das frequências de irrigação e os tratamentos com irrigação diária e a cada dois dias apresentaram receita líquida positiva. No segundo experimento as variáveis, produtividade comercial, diâmetro polar, diâmetro equatorial, teor de sólidos solúveis, espessura da polpa e firmeza da polpa sofreram influência dos tratamentos, e somente os tratamentos $32 \mathrm{~F}$ e $64 \mathrm{~F}$ apresentaram receita líquida positiva. Os melhores resultados foram obtidos com a frequência de irrigação diária. Na fertirrigação com nitrogênio, a melhor produtividade e qualidade do meloeiro foi obtida no tratamento $64 \mathrm{~F}$.

Palavras-chave: Cucumis melo, quimigação, nitrogênio.

\section{ABSTRACT \\ Management of the melon crop under different irrigation frequencies and nitrogen fertigation}

Melon is the main vegetable crop exported by the Brazilian state of Ceará, Brazil, and also an important income (and job) generator for the region. Considering this, we aimed at evaluating the effects of different irrigation frequencies and the effects of nitrogen fertigation on the physical characteristics and productivity of yellow melon. The experiment was carried out at the Cruz municipality, Ceará, from October $10^{\text {th }}$ to December $15^{\text {th }}, 2010$. We evaluated the hybrid Canarian Kobayashi under various drip irrigation regimes. On experiment I, six irrigation frequencies were evaluated: daily $(50 \%$ in the morning and $50 \%$ on the afternoon), daily ( $100 \%$ on the morning), daily ( $100 \%$ on the afternoon), every two days by morning, every three days at morning and every four days at morning. On experiment II, six nitrogen fertigation frequencies were evaluated: two $(2 \mathrm{~F})$, four (4F), eight (8F), sixteen (16F), thirty-two (32F) and sixty-four (64F) fertigations during the development cycle of the crop. Randomized block design with four replications was used. A simplified economic analysis was also carried out, for the two experiments. In the first experiment, the values of fruit weight, marketable yield, polar diameter, equatorial diameter and pulp thickness were influenced by the irrigation frequency and those who had daily irrigation and every two days recorded net positive revenue. In the second experiment the variables marketable yield, polar diameter, equatorial diameter, soluble solids, pulp thickness and pulp firmness were influenced by treatments, and only the $32 \mathrm{~F}$ and $64 \mathrm{~F}$ treatments had positive net income. The daily irrigation frequencies showed the best results. In fertigation with nitrogen, better yield and quality of melon was obtained with treatment 64F.

Keywords: Cucumis melo, chemigation, nitrogen.

(Recebido para publicação em 14 de janeiro de 2013; aceito em 21 de março de 2014) (Received on January 14, 2013; accepted on March 21, 2014)

$\mathrm{D}$ e acordo com a FAO (2009) a China é o maior produtor mundial de melão, com uma produção de 12 bilhões de toneladas, gerando uma receita bruta de US\$2.138.588.000. O Brasil aparece na $11^{\circ}$ posição, sendo o $3^{\circ}$ maior produtor da América Latina. A região Nordeste é a principal produtora de melão do Brasil, sendo os estados do Rio Grande do Norte e do Ceará aqueles que mais se destacam. Essa região possui condições climáticas específicas para o desenvolvimento adequado do meloeiro, dentre estas, podem-se citar a baixa umidade e as altas temperaturas.

O melão é a principal olerícola exportada pelo estado do Ceará. No biênio, 2008 e 2009, as exportações de melão alcançaram um total de US\$ 159,8 milhões, e no ano de 2010 este valor foi de US\$ 74,2 milhões. Em 2012, os principais destinos de venda do melão produzido no Ceará foram Holanda (44,25\%), Reino Unido (31,53\%) e
Espanha (14,59\%), ou seja, esses três países são responsáveis pela compra de mais de $90 \%$ da produção do estado (IPECE, 2013).

A água é um dos principais fatores de produção, sendo responsável pelo crescimento e desenvolvimento das plantas. A irregularidade e as baixas precipitações, características observadas na região nordeste brasileira, representam grande entrave ao desenvolvimento agrícola. Diante dessa situação, a irriga- 
ção é apresentada como uma saída para superar a escassez de água e permitir ganhos na produção (Drumond, 2003). A irrigação pode ser definida como um conjunto de operações que visam satisfazer a necessidade hídrica das culturas. (Souza \& Andrade, 2010).

Sousa et al. (1999), estudando a influência de cinco frequências de irrigação (0,5, 1, 2, 3 e 4 dias), em um solo arenoso, na cultura do meloeiro fertirrigado, verificaram que as frequências de duas vezes ao dia, diariamente e a cada dois dias apresentaram as maiores produtividades comerciais 67,$2 ; 63,9$ e 53,7 $\mathrm{t} \mathrm{ha}^{-1}$, respectivamente, sendo estas as frequências recomendadas para o cultivo em solo arenoso. Os autores não encontraram diferença significativa entre os tratamentos, com relação ao peso médio dos frutos.

Coelho et al. (1999) avaliaram em um solo arenoso coeso de tabuleiro costeiro a influência da combinação de quatro intervalos de irrigação $(1,2$, 3 e 4 dias), duas disposições de linhas laterais (uma junto à fileira de plantas e outra entre fileiras alternadas de plantas) e dois níveis de disposição do sistema de irrigação (gotejamento enterrado a $25 \mathrm{~cm}$ de profundidade e gotejamento superficial) sobre a produtividade do meloeiro. Os autores concluíram que não houve diferença significativa entre os intervalos de irrigação e a disposição das linhas laterais na produtividade do meloeiro. A produtividade comercial máxima foi de $39,6 \mathrm{tha}^{-1}$, com intervalo de irrigação de dois dias.

Para Marouelli et al. (2001), o parcelamento da dose dos nutrientes na cultura do meloeiro contribui para que a fertilidade do solo se mantenha alta durante todo o seu ciclo de desenvolvimento, maximizando assim a absorção de nutrientes pelo sistema radicular. Conceição (2008) diz que a época e a frequência de aplicação dos fertilizantes serão dependentes da curva de absorção de nutrientes da cultura, do manejo da irrigação e do sistema de irrigação empregado.

Pinto et al. (1993) avaliaram a influência de duas frequências de irrigação, diária e três vezes por semana, e oito períodos de aplicação de nitrogênio na cultura do melão, em diversas combi- nações. Esses autores verificaram que as maiores produções de frutos comerciais, 26,4 e 25,9 $\mathrm{t} \mathrm{ha}^{-1}$, foram obtidas nos tratamentos onde o nitrogênio e o potássio foram aplicados diariamente. Os autores concluíram ainda que os tratamentos não influenciaram os parâmetros qualitativos, $\mathrm{pH}$ e teor de sólidos solúveis ( ${ }^{\circ}$ Brix), dos frutos de melão.

Pinto et al. (1996) estudaram os efeitos de duas frequências de fertirrigação com nitrogênio, diária e a cada dois dias, e três períodos de aplicação, 30, 42 e 55 dias após a emergência do meloeiro. Os autores constataram que a frequência de fertirrigação diária apresentou produção de frutos comerciais, significativamente superior, a frequência de fertirrigação a cada dois dias e que o melhor período para aplicação é de até 42 dias após a emergência do meloeiro, pois a aplicação de nitrogênio próximo à colheita pode comprometer a qualidade dos frutos.

O presente trabalho objetivou avaliar os efeitos de diferentes frequências de irrigação e de fertirrigação com nitrogênio sobre as características químicas, físicas e a produtividade do melão amarelo na região Norte do estado do Ceará.

\section{MATERIAL E MÉTODOS}

O presente trabalho foi conduzido no sítio Paraguai, município de Cruz, localizado na região Norte do estado do Ceará (0254'24,55'S, 40²4’20,51'”, $19 \mathrm{~m}$ de altitude), de 10 de outubro a 15 de dezembro de 2010.

De acordo com a classificação de Köppen, o clima da região é do tipo Aw', caracterizado como clima tropical chuvoso, onde a época mais seca ocorre no inverno e o ápice de chuvas no outono, com precipitação média anual de 960 mm e evapotranspiração média anual de $1.600 \mathrm{~mm}$. Apresenta temperaturas mínima, média e máxima anuais de 23 , 28 e $35^{\circ} \mathrm{C}$, respectivamente. A região possui insolação média anual de 3.000 horas, umidade relativa média anual de $70 \%$ e velocidade média do vento de $2,10 \mathrm{~m} \mathrm{~s}^{-1}$.

O solo foi classificado como Neossolo Quartzarênico e apresentava as seguintes propriedades físicas na camada de $0-20 \mathrm{~cm}$ : areia fina $=248$ $\mathrm{g} \mathrm{kg}^{-1}$; areia grossa $=675 \mathrm{~g} \mathrm{~kg}^{-1}$; silte $=36 \mathrm{~g} \mathrm{~kg}^{-1}$; argila $=41 \mathrm{~g} \mathrm{~kg}^{-1}$; massa específica $=1,52 \mathrm{~g} \mathrm{~cm}^{-3}$; e água útil $=$

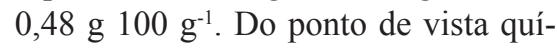
mico, o solo apresentava as seguintes características: $\mathrm{pH}=4,9 ; \mathrm{Al}, \mathrm{Ca}, \mathrm{Mg}$ e $\mathrm{K}$, respectivamente 0,$2 ; 0,8 ; 0,6$ e 0,1 $\mathrm{cmol}_{\mathrm{c}} \mathrm{dm}^{-3}$ e $\mathrm{P}=2 \mathrm{mg} \mathrm{dm}^{-3}$.

O preparo do solo consistiu em desmatamento, seguido da retirada dos troncos e galhos mais grossos, incorporação do restante da matéria orgânica (folhas e galhos mais finos), sistematização e gradagem cruzada.

Avaliou-se o melão tipo amarelo (Cucumis melo), híbrido Canarian Kobayashi. Este foi semeado diretamente em campo no dia 10 de outubro de 2010, seguindo o espaçamento de 2,0 x 0,5 m. Para garantir a uniformidade de germinação do estande, foram colocadas duas sementes por cova.

Durante a condução dos experimentos, foram realizados tratos culturais de desbaste das plântulas, capinas, monitoramento do estado fitossanitário e nutricional, pulverizações, viragem e desbaste dos frutos com má formação e colheita.

O sistema de irrigação instalado na área experimental foi do tipo localizado, por gotejamento superficial, sendo composto por mangueiras gotejadoras com diâmetro de 16 mm, distribuídas paralelamente à linha de plantio, sendo uma linha de gotejo por linha de plantio. Estas apresentavam gotejadores integrados de fluxo turbulento, espaçados de $0,40 \mathrm{~m}$ entre si, com vazão individual de $1,60 \mathrm{~L} \mathrm{~h}^{-1}$ à pressão nominal de 10 mca.

Foram realizados dois experimentos; no primeiro foram avaliadas seis frequências de irrigação e no segundo foram avaliadas seis frequências de fertirrigação com nitrogênio. O delineamento experimental empregado foi de blocos ao acaso, com quatro repetições, para ambos os experimentos.

No experimento I foram avaliadas diferentes frequências de rega e períodos de aplicação: diariamente nos períodos da manhã e da tarde (1DMT); diariamente no período da manhã (1DM); diariamente no período da tarde (1DT); a cada dois dias no período da manhã (2DM); a cada três dias no período da 
manhã (3DM) e a cada quatro dias no período da manhã (4DM). Nos tratamentos 2DM, 3DM e 4DM, a quantidade de água aplicada correspondeu ao somatório da evapotranspiração da cultura no período, ou seja, o acumulado de 2, 3 e 4 dias, respectivamente.

No experimento II foram realizadas duas $(2 \mathrm{~F})$; quatro $(4 \mathrm{~F})$; oito $(8 \mathrm{~F})$; dezesseis $(16 \mathrm{~F})$; trinta e duas $(32 \mathrm{~F})$ e sessenta e quatro (64F) fertirrigações durante o ciclo da cultura. Para o tratamento com fertirrigação diária (64F), a dose de nitrogênio a ser aplicada na semana era dividida por sete, e para os demais tratamentos a quantidade de fertilizante aplicada em cada fertirrigação era proporcional ao número de dias entre duas aplicações consecutivas. A lâmina de água foi aplicada diariamente para todos os tratamentos.

A dose dos fertilizantes foi definida com base na análise de solo e nas recomendações propostas por Crisóstomo et al. (2002), as quais preconizam a aplicação dos principais nutrientes de acordo com a marcha de absorção da cultura. O parcelamento dos nutrientes na fertirrigação foi feito conforme: $10 \%$ por semana entre a $1^{\mathrm{a}}$ e a $4^{\mathrm{a}}$ semana, $15 \%$ por semana na $5^{\mathrm{a}}$ e na $6^{\mathrm{a}}$ semana e $10 \%$ por semana entre a $7^{\mathrm{a}}$ e a $9^{\mathrm{a}}$ semana.

Empregou-se os fertilizantes uréia (120 $\left.\mathrm{kg} \mathrm{ha}^{-1} \mathrm{de} \mathrm{N}\right)$, nitrato de cálcio (68 $\left.\mathrm{kg} \mathrm{ha}^{-1} \mathrm{de} \mathrm{Ca}\right)$, ácido fosfórico $(240 \mathrm{~kg}$ $\mathrm{ha}^{-1}$ de $\left.\mathrm{P}\right)$, cloreto de potássio branco (300 $\mathrm{kg} \mathrm{ha}^{-1}$ de $\mathrm{K}$ ), sulfato de magnésio (23 $\mathrm{kg} \mathrm{ha}^{-1}$ de $\mathrm{Mg}$ ), ácido bórico (1 g planta $^{-1}$ de B) e sulfato de zinco (2 g planta $^{-1}$ de Zn). Todos os fertilizantes foram aplicados via fertirrigação e parcelados de acordo com Crisóstomo et al. (2002); apenas o nitrogênio, no experimento II, com diferentes frequências de fertirrigação, foi parcelado de acordo com os tratamentos. Na determinação da dose de nitrogênio empregada na fertirrigação, foi considerada também a quantidade de $\mathrm{N}$ disponibilizada pelo nitrato de cálcio $\left(55,5 \mathrm{~kg} \mathrm{ha}^{-1}\right)$. A quantidade dos outros fertilizantes, utilizados na fertirrigação, foi aplicada de uma única vez durante o período considerado, por exemplo, no caso do potássio, a quantidade de $10 \%$ na primeira semana foi aplicada de uma única vez. Para a dissolução e mistura dos adubos, foram utilizados volumes de calda equivalentes a 30 L. Esse volume foi suficiente para a injeção da solução nutritiva. A taxa de injeção do sistema era de $90 \mathrm{~L} \mathrm{~h}^{-1}$.

Em cada um dos quatro blocos experimentais, havia seis parcelas com área individual de $70 \mathrm{~m}^{2}(35 \times 2 \mathrm{~m})$ contendo 70 plantas, totalizando, dessa forma, 24 unidades experimentais. Para as variáveis relacionadas com a produtividade, foram utilizados todos os frutos das quatro plantas úteis de cada parcela; a $5^{\mathrm{a}} ; 25^{\mathrm{a}} ; 45^{\mathrm{a}}$ e a $60^{\mathrm{a}}$ plantas, de forma que o restante foi considerado como bordadura. Para as variáveis qualitativas, foram selecionados ao acaso dois frutos do total colhido das quatro plantas úteis. A aplicação dos tratamentos foi iniciada em 10 de outubro e finalizada em 15 de dezembro de 2010. Os dois experimentos foram realizados ao mesmo tempo em áreas vizinhas.

A colheita foi realizada aos 65 dias após o plantio (DAP) para o experimento II, com frequências de fertirrigação, e aos 66 (DAP) para o experimento I, com frequências de irrigação. A colheita foi iniciada quando todos os frutos apresentavam coloração amarela e características apropriadas à comercialização.

Os frutos foram avaliados quanto à produtividade comercial (PC), massa dos frutos (MF), diâmetros polar (DP) e equatorial (DE), espessuras da casca (EC) e da polpa (EP), teor de sólidos solúveis (SS) e firmeza da polpa (FP). A PC ( $\left.\mathrm{t} \mathrm{ha}^{-1}\right)$ e a MF $(\mathrm{kg})$ foram obtidas através de uma balança de precisão Adventurer ${ }^{\mathrm{TM}}$, enquanto que DP (cm), $\mathrm{DE}(\mathrm{cm}), \mathrm{EC}(\mathrm{cm})$ e EP $(\mathrm{cm})$ foram medidos com um paquímetro digital Digimess $^{\odot}$. O teor de SS ( ${ }^{\circ}$ Brix) e a FP (Newton) foram determinados, respectivamente, por um refratômetro portátil analógico RT-280 Instrutemp ${ }^{\odot}$ (escala de 0 a $80 \%$ e resolução de $1 \%{ }^{\circ}$ Brix) e um penetrômetro Fruit Test ${ }^{\mathrm{TM}}$ FT30 (acurácia de $1^{0}$ ), sendo que os valores fornecidos por este último instrumento foram posteriormente transformados para Newton.

De posse das produtividades de todos os tratamentos, calculou-se a receita líquida para os mesmos utilizando a planilha eletrônica do Instituto Agropolos, adaptada de Souza (2006), a fim de se verificar o retorno econômico para o produtor.

Primeiramente calculou-se o custo cultural (CC) pela fórmula:

$\mathrm{CC}=\mathrm{SE}+\mathrm{FE}+\mathrm{DE}+\mathrm{EE}+\mathrm{OME}+\mathrm{OMA}$

Onde $\mathrm{SE}=$ sementes; $\mathrm{FE}=$ fertilizantes; $\mathrm{DE}=$ defensivos; $\mathrm{EE}=$ energia elétrica; $\mathrm{OME}=$ operação mecanizada; OMA= operação manual (todos obtidos em R\$).

Posteriormente, calculou-se o custo total (CT):

$$
\mathrm{CT}=\mathrm{CC}+\mathrm{CA}+\mathrm{EI}+\mathrm{JC}
$$

Onde $\mathrm{CC}=$ custo cultural; $\mathrm{CA}=$ custos administrativos; $\mathrm{EI}=$ equipamento de irrigação; JC= juros sob custeio/ano (todos obtidos em R\$).

Por fim, calculou-se a receita bruta $(\mathrm{RB})$ e a receita líquida $(\mathrm{RL})$ :

$$
\begin{gathered}
\mathrm{RB}=\mathrm{PT} * \mathrm{PREÇO} \\
\mathrm{RL}=\mathrm{RB}-\mathrm{CT}
\end{gathered}
$$

Onde $\mathrm{PT}=$ produtividade total estimada $\left(\mathrm{kg} \mathrm{ha}^{-1}\right) ; \mathrm{PREÇO}=$ preço $(\mathrm{R} \$$ $\left.\mathrm{kg}^{-1}\right) ; \mathrm{CT}=$ custo total $(\mathrm{R} \$)$

Os dados obtidos foram submetidos à análise de variância (teste F), ao nível de $5 \%$ de probabilidade. Para os tratamentos qualitativos foi utilizado o teste de Tukey a 5\% de probabilidade. Já os tratamentos quantitativos foram submetidos à análise de regressão.

\section{RESULTADOS E DISCUSSÃO}

Experimento I (frequências de irrigação) - As variáveis massa dos frutos (MF), produtividade comercial (PC), diâmetro polar (DP), diâmetro equatorial (DE) e espessura da polpa (EP) sofreram influência das frequências de irrigação. Já as variáveis, teor de sólidos solúveis (SS), espessura da casca (EC) e firmeza da polpa (FP) não apresentaram diferença significativa, ao nível de $5 \%$ de probabilidade, para os tratamentos empregados.

$\mathrm{Na}$ Tabela 1, encontram-se os valores médios das variáveis estudadas em função das frequências de irrigação. De maneira geral, as frequências de irrigação 1DM, 1DT e 1DMT apresentaram os melhores valores para todas as variáveis estudadas, e os tratamentos 3DM e 4DM os valores mais baixos. 
Tabela 1. Valores médios de massa dos frutos (MF), produtividade comercial (PC), diâmetro polar (DP), diâmetro equatorial (DE), espessura da polpa (EP), sólidos solúveis (SS), espessura da casca (EC) e firmeza da polpa (FP) dos frutos do meloeiro em função das frequências de irrigação (mean values of fruit weight (MF), marketable yield (PC), polar diameter (DP), equatorial diameter (DE), pulp thickness (EP), soluble solids (SS), rind thickness (EC), and pulp firmness (FP) of the fruits of melon plants depending on irrigation frequency). Cruz, UFC, 2010.

\begin{tabular}{llcllcccc}
\hline Tratamento & $\mathbf{M F}(\mathbf{k g})$ & $\mathbf{P C}(\mathbf{t} / \mathbf{h a})$ & $\mathbf{D P}(\mathbf{c m})$ & $\mathbf{D E} \mathbf{( c m})$ & $\mathbf{E P}(\mathbf{c m})$ & $\mathbf{S S}\left({ }^{\circ} \mathbf{B r i x}\right)$ & $\mathbf{E C}(\mathbf{c m})$ & $\mathbf{F P}(\mathbf{N})$ \\
\hline 1DM & $2,0 \mathrm{~A}$ & $44,7 \mathrm{~A}$ & $15,4 \mathrm{~A}$ & $15,7 \mathrm{AB}$ & $4,3 \mathrm{~A}$ & $12,4 \mathrm{~A}$ & $0,4 \mathrm{~A}$ & $44,0 \mathrm{~A}$ \\
1DT & $1,9 \mathrm{AB}$ & $42,8 \mathrm{~A}$ & $15,2 \mathrm{~A}$ & $15,7 \mathrm{AB}$ & $4,0 \mathrm{AB}$ & $12,4 \mathrm{~A}$ & $0,4 \mathrm{~A}$ & $42,6 \mathrm{~A}$ \\
1DMT & $2,0 \mathrm{~A}$ & $54,4 \mathrm{~A}$ & $15,2 \mathrm{~A}$ & $16,2 \mathrm{~A}$ & $4,2 \mathrm{~A}$ & $12,5 \mathrm{~A}$ & $0,4 \mathrm{~A}$ & $43,0 \mathrm{~A}$ \\
2DM & $1,5 \mathrm{ABC}$ & $23,8 \mathrm{~B}$ & $14,1 \mathrm{AB}$ & $14.3 \mathrm{ABC}$ & $3,2 \mathrm{~B}$ & $12,5 \mathrm{~A}$ & $0,4 \mathrm{~A}$ & $41,3 \mathrm{~A}$ \\
3DM & $1,2 \mathrm{C}$ & $21,4 \mathrm{~B}$ & $12,9 \mathrm{~B}$ & $13,1 \mathrm{C}$ & $3,2 \mathrm{~B}$ & $13,1 \mathrm{~A}$ & $0,3 \mathrm{~A}$ & $41,0 \mathrm{~A}$ \\
4DM & $1,4 \mathrm{BC}$ & $25,5 \mathrm{~B}$ & $13,5 \mathrm{AB}$ & $14,1 \mathrm{BC}$ & $3,4 \mathrm{AB}$ & $13,0 \mathrm{~A}$ & $0,4 \mathrm{~A}$ & $43,6 \mathrm{~A}$ \\
\hline F & 8,4 & 19 & 6 & 8,2 & 6,6 & 0,8 & 0,3 & 0,7 \\
CV (\%) & 11,4 & 19,2 & 4 & 3 & 9,4 & 5,11 & 33,7 & 7 \\
\hline
\end{tabular}

Médias seguidas da mesma letra na coluna, não diferem significativamente entre si pelo teste de Tukey a 5\% de probabilidade [means followed by the same letter are not significantly different by Tukey test $(5 \%)$ probability]; Frequências de irrigação: $1 \mathrm{DM}=100 \%$ pela manhã; $1 \mathrm{DT}=100 \%$ de tarde; $1 \mathrm{DMT}=50 \%$ de manhã e $50 \%$ de tarde; $2 \mathrm{DM}=$ a cada dois dias pela manhã; $3 \mathrm{DM}=\mathrm{a}$ cada três dias pela manhã; 4DM = a cada quatro dias pela manhã. (irrigation frequency: $1 \mathrm{DM}=100 \%$ on the morning; $1 \mathrm{DA}=100 \%$ on the afternoon; $1 \mathrm{DMA}$ $=50 \%$ in the morning and $50 \%$ on the afternoon; $2 \mathrm{DM}=$ every two days at morning; $3 \mathrm{DM}$ every three days at morning; $4 \mathrm{DM}=$ every four days in the morning).

Para a produtividade comercial, os tratamentos 1DM, 1DT e 1DMT apresentaram os maiores valores, não

diferindo entre si, e diferindo dos demais tratamentos. Já para os diâmetros polar e equatorial, os maiores valores foram

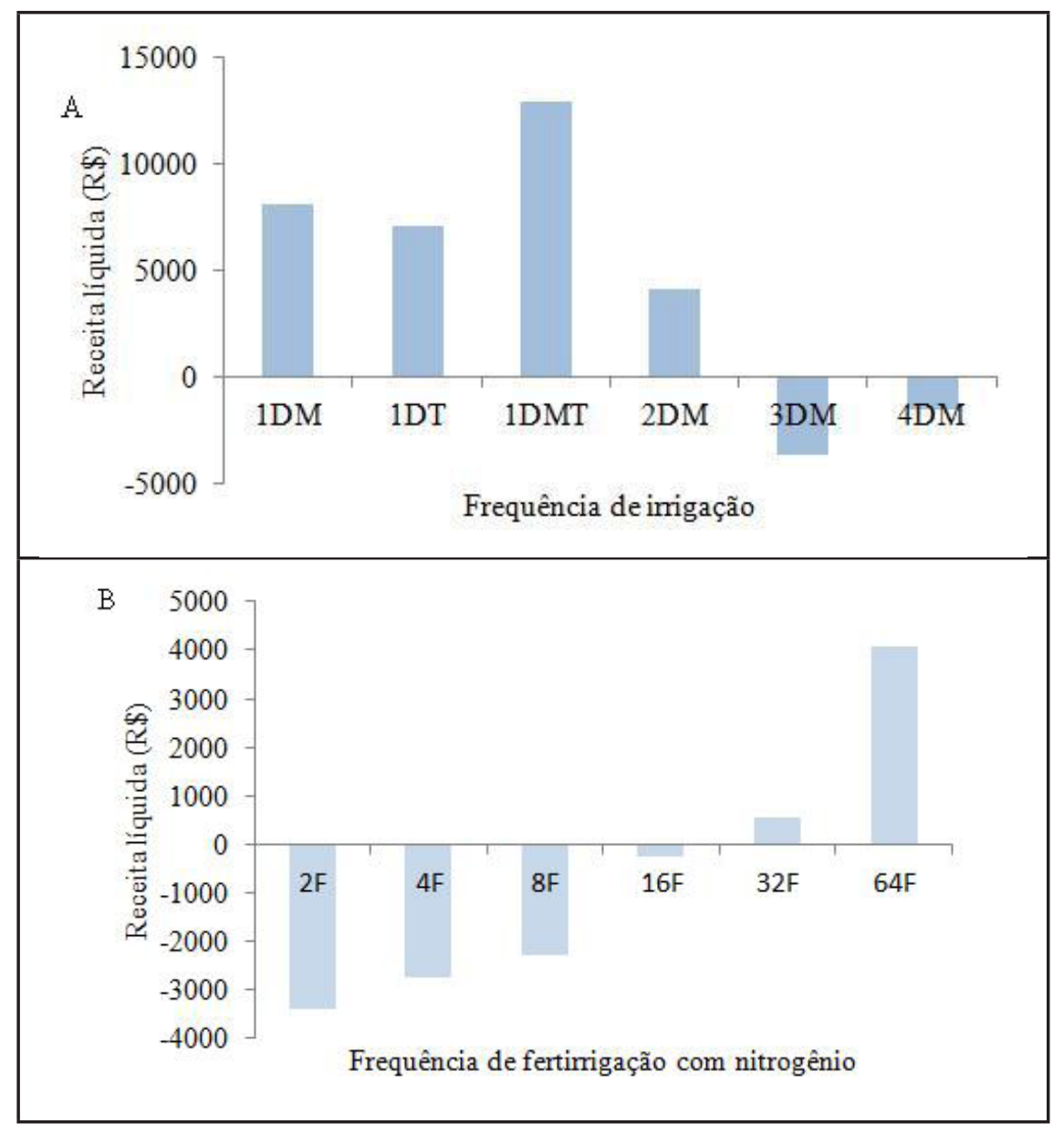

Figura 1. A) receita líquida, em Reais, da produtividade do melão amarelo em função das frequências de irrigação (net income in Reais, depending on the productivity of yellow melon and the irrigation frequency); B) em função do número de fertirrigações com nitrogênio (depending on the number of fertigations with nitrogen). Cruz, UFC, 2010. observados nos tratamentos 1DM, 1DT, 1DMT e 2DM, sendo que o tratamento 2DM não diferiu do 4DM, quanto ao diâmetro polar. Para a massa dos frutos e a espessura da polpa, os maiores valores foram obtidos com os tratamentos 1DM, 1DT e 1DMT. Entretanto, para a massa dos frutos esses tratamentos não diferiram do 2DM, e para a espessura da polpa eles não diferiram do 4DM.

Pereira Filho (2012), testando seis frequências de irrigação na cultura do melão em Pentecoste-CE: F1, irrigação realizada duas vezes ao dia (manhã e tarde); F2, irrigação diária somente pela manhã; F3, irrigação realizada a cada dois dias; F4, irrigação realizada a cada três dias; F5, irrigação realizada a cada quatro dias e F6, irrigação realizada a cada cindo dias, observaram que a maior produtividade $\left(18,8 \mathrm{tha}^{-1}\right)$, foi obtida no tratamento $F 2$, sendo que esse valor não diferiu estatisticamente daqueles obtidos nos tratamentos $\mathrm{F} 1$ e F3; já o maior valor medido para a variável massa dos frutos foi de $1,1 \mathrm{~kg}$ fruto $^{-1}$, no tratamento $\mathrm{F} 1$. Com relação aos diâmetros polar e equatorial, os maiores valores, 12,3 e 15,2 $\mathrm{cm}$, foram medidos, respectivamente, nos tratamentos F2 e F1.

Os resultados encontrados podem ser explicados pelo fato dos solos arenosos não reterem água de forma eficiente, pois possuem baixa quantidade de microporos; com isso, quando se aplica uma lâmina de água acumulada, 


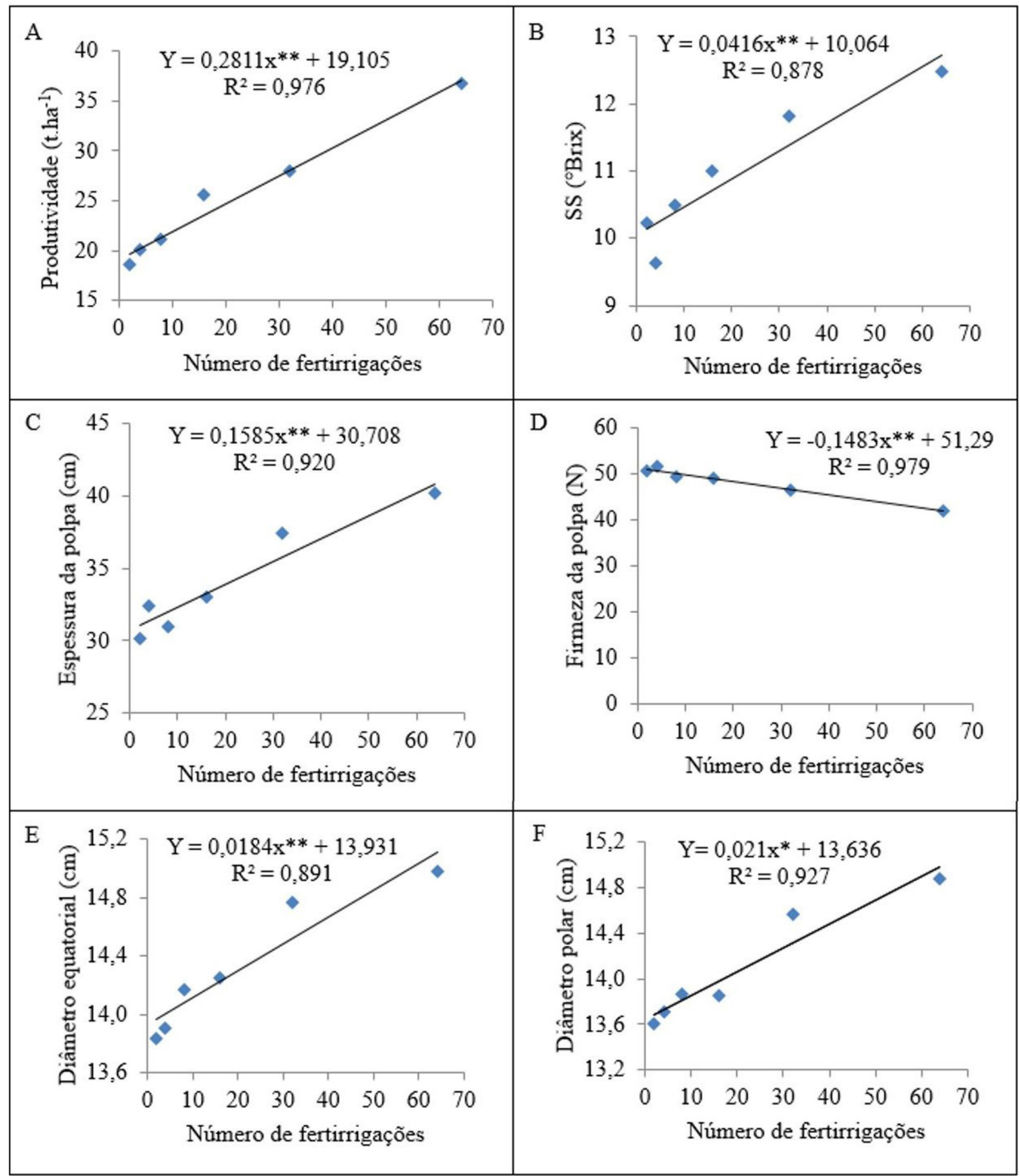

Figura 2. A) produtividade comercial (marketable yield); B) teor de sólidos solúveis (soluble solids); C) espessura da polpa (pulp thickness); D) firmeza da polpa (pulp firmness); E) diâmetro equatorial (equatorial diameter); F) diâmetro polar (polar diameter) dos frutos do meloeiro em função do número de fertirrigações com nitrogênio (of melon fruits depending on number of fertigations with nitrogen). Cruz, UFC, 2010.

grande parte desta pode ser perdida por drenagem. Já, quando a lâmina de água é fornecida diariamente, a planta recebe um fornecimento mais regular de água, ocasionando assim, suprimento hídrico adequado. Dessa forma, os problemas com déficit hídrico serão menores.

Na Figura 1A estão os valores de receita líquida, obtida da análise econômica simplificada, em função das frequências de irrigação. Nota-se que apenas os tratamentos com fertirrigação diária, $1 \mathrm{DM}, 1 \mathrm{DT}, 1 \mathrm{DMT}$, e o tratamento $2 \mathrm{MD}$ apresentaram lucro para o produtor. $\mathrm{O}$ tratamento $1 \mathrm{DMT}$ 
apresentou a maior receita líquida positiva ( $\left.\mathrm{R} \$ 12.989,00 \mathrm{ha}^{-1}\right)$, e o tratamento que apresentou a menor receita líquida foi o 3DM, com valor negativo de $\mathrm{R} \$$ $3.627,88 \mathrm{ha}^{-1}$.

Experimento II (frequências de fertirrigação com nitrogênio) - As variáveis, produtividade comercial (PC), diâmetro polar (DP), diâmetro equatorial (DE), teor de sólidos solúveis (SS), espessura da polpa (EP) e firmeza da polpa (FP) sofreram influência das frequências de fertirrigação com nitrogênio, ao nível de 5\% de probabilidade. Já as variáveis massa dos frutos (MF) e espessura da casca (EC) não apresentaram diferença estatística para os tratamentos empregados.

A produtividade comercial aumentou linearmente com o maior parcelamento da dose de nitrogênio (Figura 2A). Segundo Crisóstomo et al. (2002), o nitrogênio aumenta o peso e o número de frutos. De acordo com Nerson et al. (1992), uma maior absorção de nitrogênio pelo meloeiro acarretará em aumento na produção de fotoassimilados, e consequentemente, elevação na produtividade da cultura.

O teor de sólidos solúveis totais aumentou de forma linear com o aumento da frequência de aplicação da dose de nitrogênio (Figura 2B). Segundo Crisóstomo et al. (2002), o nitrogênio exerce influência sobre o conteúdo do sólidos solúveis totais do meloeiro e na absorção do potássio.

A espessura da polpa aumentou de forma linear quando da maior frequência de aplicação da dose de nitrogênio (Figura 2C). Segundo Carmello (1999), o nitrogênio produz alterações morfofisiológicas na planta que influenciam a fotossíntese, o desenvolvimento e atividades do sistema radicular, a absorção iônica de nutrientes, além de processos de crescimento e diferenciação celular.

Nota-se que a firmeza da polpa diminuiu de forma linear com o aumento da frequência de aplicação da dose de nitrogênio (Figura 2D). Ou seja, o fornecimento adequado de água e nitrogênio favoreceu o crescimento vegetativo das plantas. Dessa forma, as células dos tecidos do fruto tendem a se manter mais turgidas, proporcionando assim uma polpa menos firme.
Percebe-se que, tanto o diâmetro equatorial quanto o polar, aumentaram de forma linear com o aumento da frequência de aplicação da dose de nitrogênio (Figura 2E e 2F). Ou seja, com a maior disponibilidade de nitrogênio, o meloeiro produzirá frutos maiores. Queiroga et al. (2007) afirmam que o nitrogênio pode proporcionar uma maior produção de fotoassimilados para os frutos, favorecendo assim, o crescimento desses.

Pereira Filho (2012), avaliando quatro aplicações parceladas da dose recomendada de nitrogênio, $90 \mathrm{~kg} \mathrm{ha-1}$ [(100\% aos 20 dias após a semeadura (DAS)]; 30\% na semeadura e 70\% aos 20 DAS; $30 \%$ na semeadura, 30\% aos 20 dias e $40 \%$ aos 35 DAS; $20 \%$ na semeadura, 30\% aos 20 dias, $30 \%$ aos 35 dias e $20 \%$ aos 50 DAS) na cultura do meloeiro no Vale do Curu-CE, concluíram que não houve diferença estatística entre os tratamentos para a variável produtividade, diferindo dos resultados encontrados no presente trabalho, sendo que o maior valor, $15,4 \mathrm{t} \mathrm{ha}^{-1}$, foi encontrado no tratamento (30-30-40-0). Já a massa dos frutos, os diâmetros polar e equatorial, foram influenciados pelos tratamentos, sendo os maiores valores para essas variáveis, $0,93 \mathrm{~kg}$ fruto $^{-1}, 14,1$ $\mathrm{cm}$ e $12,3 \mathrm{~cm}$ foram obtidos, respectivamente, nos tratamentos (30-30-40-0) e (20-30-30-20). Ainda, segundo o autor, a variável teor de sólidos solúveis (SS) não foi influenciada pelos tratamentos.

Os resultados obtidos nesse experimento podem ser explicados pelo fato de que, com um maior parcelamento da dose de nitrogênio, as perdas por lixiviação e volatilização são reduzidas; com isso, a cultura tem maior aproveitamento desse nutriente durante todo o seu ciclo fenológico.

Na Figura 1B está o resultado da receita líquida, obtida da análise econômica simplificada, em função do número de fertirrigações com nitrogênio. Apenas os tratamentos $32 \mathrm{~F}$ e $64 \mathrm{~F}$ apresentaram lucro, ou seja, uma receita líquida positiva para o produtor. $\mathrm{O}$ tratamento $32 \mathrm{~F}$ apresentou valor positivo de apenas $\mathrm{R} \$$ $548,36 \mathrm{ha}^{-1}$ e o tratamento $64 \mathrm{~F}$ foi o que proporcionou a maior receita líquida para o produtor, com valor positivo de $\mathrm{R} \$ 4.058,12$ ha $^{-1}$. Já o tratamento que apresentou a maior receita líquida negativa foi o $2 \mathrm{~F}$ de $\mathrm{R} \$ 3.403,86 \mathrm{ha}^{-1}$.

Portanto, a frequência de irrigação interferiu no desenvolvimento da cultura do meloeiro, sendo a frequência diária, em qualquer período de aplicação ou dividida entre o período da manhã e da tarde, indicada para o melhor aproveitamento dos recursos hídricos e maior rendimento econômico, nas condições de estudo. O parcelamento da aplicação do nitrogênio interferiu na eficiência do suprimento deste nutriente para a planta. Com isso, a frequência de fertirrigação indicada para o manejo mais eficiente e que apresenta a melhor rentabilidade para o produtor, foi a de 64 fertirrigações ao longo do ciclo do meloeiro, ou seja, diariamente.

\section{REFERÊNCIAS}

CARMELLO QAC. 1999. Curso de nutrição/ fertirrigação na irrigação localizada. Piracicaba: ESALQ, 59 p.

COELHO EF; SOUSA VF; RODRIGUES BHN; SOUZA VAB; ANDRADE CLT. 1999. Produtividade do meloeiro sob diferentes intervalos de irrigação e disposições de linhas laterais de gotejamento em solo arenoso coeso. Revista Brasileira de Engenharia Agrícola e Ambiental 03: 309-315.

CONCEIÇÃO MAF. 2008. A irrigação na produção de uvas para elaboração de vinhos finos. Bento Gonçalves: EMBRAPA-CNPUV, 20p. (Circular técnica, 79).

CRISÓSTOMO LA; SANTOS AA; RAIJ B; FARIA CMB; SILVA DJ; FERNANDES FAM; SANTOS FJS; CRISÓSTOMO JR; FREITAS JAD; HOLANDO JS; CARDOZO JW; COSTA ND. 2002. Adubação, irrigação, híbridos e práticas culturais para o meloeiro no Nordeste. Fortaleza: EMBRAPA AGROINDÚSTRIA TROPICAL, $21 \mathrm{p}$. (Circular técnica, 14).

DRUMOND LCD. 2003. Aplicação de água residuária de suinocultura por aspersão em malha: desempenho hidráulico e produção de matéria seca de Tifton. Jaboticabal: UNESP. $102 \mathrm{f}$. (Tese doutorado).

FAO. 2009. Faostat database results. Disponível em: http://faostat.fao.org/faostat/servlet. Acesso em: 28 mai. 2011.

IPECE. 2013. Enfoque Econômico. Disponível em: www.ipece.ce.gov.br. Acessado em: 05 dez. 2013.

MAROUELLI WA; MEDEIROS JF; SILVA HR; PINTO JM; SILVA WLC. 2001. Irrigação e fertirrigação do meloeiro por gotejamento. Brasília: EMBRAPA-CNPH. 28p. (Circular Técnica 25).

NERSON H; PARIS HS; EDELSTEIN M. 
1992. Nitrogen and phosphorus stress repair muskmelon (Cucumis melo) seedlings. Journal of Plant Nutrition 10: 1835-1841.

PEREIRA FILHO JV. 2012. Cultivo do melão sob diferentes frequências de irrigação e parcelamento da adubação nitrogenada no Vale do Curu, CE. Fortaleza: UFC. 95 p. (Dissertação mestrado).

PINTO JM; SOARES JM; CHOUDHURY EN; PEREIRA JR. 1993. Adubação via água de irrigação na cultura do melão. Pesquisa Agropecuária Brasileira 28: 1263-1268.
PINTO JM; SOARES JM; PEREIRA JR; COSTA ND; BRITO LTL; FARIA CMB; MACIEL JL. 1996. Sistema de cultivo de melão com aplicação de fertilizantes via água de irrigação. Brasília: EMBRAPA-SPI. 24p. (Circular técnica 36).

QUEIROGA RCF; PUIATTI M; FONTES PCR; CECON PR; FINGER FR. 2007. Influência de doses de nitrogênio na produtividade e qualidade do melão Cantalupensis sob ambiente protegido. Horticultura Brasileira 25: $550-556$.
SOUSA VF; COÊLHO EF; SOUZA VAB. 1999. Frequência de irrigação em meloeiro cultivado em solo arenoso. Pesquisa Agropecuária Brasileira 4: 659-654.

SOUZA DLR. 2006. Estudo das vantagens competitivas do melão no Ceará. Fortaleza: Instituto Agropólos. 56 p.

SOUZA IH; ANDRADE EM. 2010. Manejo da irrigação. In: Semiárido e o manejo dos recursos naturais: uma proposta de uso adequado do capital natural. Fortaleza: Imprensa Universitária. Cap. 11, p. 240. 